\title{
Submerged Glow-Discharge Plasma: An Economical Approach to Convert Construction Scrap Metal into Nanomaterials
}

\author{
Peter Nai Yuh Yek $^{1 *}$, Muhammad Rafiq Mirza Julaihi ${ }^{2}$, Mohammad Shahril Osman ${ }^{1}$, Tung \\ Chuan Tiong ${ }^{1}$, Wak Ha Lee $^{3}$, and Chern Leing Lee ${ }^{4}$ \\ ${ }^{1}$ Department of Engineering, University College of Technology Sarawak, 96000, Sibu, Sarawak, \\ Malaysia \\ ${ }^{2}$ Faculty of Engineering, Swinburne University of Technology (Sarawak Campus), Jalan Simpang \\ Tiga 93350 Kuching, Sarawak, Malaysia \\ ${ }^{3}$ Faculty of Resource Science and Technology, Universiti Malaysia Sarawak (UNIMAS), 94300 \\ Kota Samarahan, Sarawak, Malaysia \\ ${ }^{4}$ Chemical Engineering Discipline, Monash University Malaysia, Jalan Lagoon Selatan, 47500, \\ Bandar Sunway, Selangor, Malaysia
}

\begin{abstract}
Submerged glow-discharge plasma (SGDP) is relatively new among the various methods available for nanomaterials synthesis (NMs) techniques. This method allows great control over the production cost of nanomaterials synthesis. A lab-scale batch type SDGP technology has been constructed to produce nanomaterials and investigate the inter-relationship between plasma excitation voltages, electrodes submerged areas and electrolyte concentration. Metal oxide nanospheres has been synthesised from different electrolyte concentrations (1M-0.001M) and characterized by Scanning Electron Microscopy (SEM) and Energy Dispersive Spectroscopy (EDS). As the major results showed that the nanospheres are uniformly spherical with diameter size distribution are between $100 \mathrm{~nm}-2 \mu \mathrm{m}$. EDS analysis shown the nano-Iron Oxide have been formed. Scrap metal initially showed around $6.45 \%$ and $93.55 \%$ of Carbon and Iron composition respectively. After SGDP process to the scrap metal, Carbon content has increased to $34-35 \%$ and Iron content has reduced to around $15-40 \%$. EDS results also shown the higher percentage of Iron amount has remained with lower electrolyte concentration and Current is proportionally related to submersion area of cathode.
\end{abstract}

\section{Introduction}

Construction is one of the most important contributors to overall socio-economic

\footnotetext{
*Corresponding author: peter.yek@ucts.edu.my
} 
development. However, without proper construction waste management, it is also a significant polluter to the environment. Over the last two decades Malaysia construction industry contributed between 3 to 5 percent of the aggregate economy of gross domestic product (GDP) [1]. The construction industry also cause abundant of construction waste and facing challenges in effective management to minimize the environmental pollution [2]. Currently, common practice in construction by developers are 3R (Reduce, Reuse, Recycle) [2, 3]. Waste left over in construction site often contains bulky materials including concrete, wood, metals, bricks, glass, plastics and so on [4]. Metals such as aluminium, copper, and steel are widely being utilized in the construction industries. Among all type of construction waste generated, $1 \%$ is consist of scrap metal products and $0.27 \%$ of scrap metal can be reused on site [5] and other sold to scrap dealers. Reusing scrap metal on site and re-melting are commonly used as profitable ways and to maintain the life cycle of metallic objects. This research explores the alternative way of converting scrap metal into higher added value product, namely nanomaterials via submerged glowdischarge plasma (SGDP).

Plasma is generated from the ionization of neutral gases by applying extra heat or energy to free the electron from the covalent bond. The plasma contains an equal number of positive ions and free negative electrons to create a strong electric field. Furthermore, glow discharge plasma in liquid also generates active radicals, an effective reducing and oxidation agents to treat biological and chemical matter [6]. Currently, glow-discharge plasma in liquid has provide new scientific challenges and emerging plasma technology widely applied in drinking and waste water treatment [7-9], hydrogen peroxide production $[10,11]$, plasma medicine [12], surface engineering [13], nanomaterial synthesis [14-17] etc.

The interest of nanomaterials synthesis via SGDP is growing due to its applicability in the industry for large scale [15]. Submerged glow-discharge plasma has been researched as an innovative and economical method for nanoparticles synthesis [14, 17-19] and offers several advantages: (1) relatively simple in experiment setup and mass production , (2) no external gas supply is needed, and (3) easily available precursor of nanoparticles [20]. The field of nanomaterials synthesis via submerged glow-discharge plasma $[14,18]$ has grown significantly due to the growing demand for various types of nanotechnology developments. This paper discusses the generation mechanism of glow discharge plasma for the preparing nanoparticles from scrap metal submerged in liquid and physical characteristics of the produced nanoparticles.

\section{Materials and methods}

\subsection{Precursor preparation}

Scrap metal of iron nail in $3 \mathrm{~mm}$ diameter was collected from a construction site then washed by distilled water to remove all the impurities attached on the scrap metal surface.

\subsection{Submerged glow-discharge plasma setup}

Fig. 1 shows the schematic diagram of SGDP experimental prototype with batch type reaction shown in using $500 \mathrm{ml}$ open top cylindrical funnel as SGDP reactor. During the plasma reaction, electrolyte has to be filled continuously to maintain the submerged area of cathode in SGDP reactor. Hence, nanospheres as product of plasma discharge will precipitate at the bottom and discharged for further purification process. SGDP reactor was supplied with electricity by using DC power supply (Kikusui, Tokyo, Japan) used to 
perform the plasma generation. A $300 \mathrm{~mm}$ platinum wire with $0.3 \mathrm{~mm}$ diameter and 99.98 mass\% purity (Nilaco, Tokyo, Japan) used as the anode; this wire was bent into a spiral along the length of a glass rod. Meanwhile, a scrap metal nail of $3 \mathrm{~mm}$ diameter used as cathode. The distance between the electrodes was kept at $30 \mathrm{~mm}$. The potassium carbonate $\left(\mathrm{K}_{2} \mathrm{CO}_{3}\right)$ used as electrolytes with the concentrations ranging from 1 to $0.01 \mathrm{~mol}$. The voltage was applied at a rate of $5 \mathrm{~V} \mathrm{~s}^{-1}$ until the formation of plasma and voltage will fixed at a constant value for $60 \mathrm{~min}$. After the plasma discharge experiments, molten droplets of nanomaterial condensed and washed with distilled water. Finally, the nanospheres were dried in oven with $105^{\circ} \mathrm{C}$ for $24 \mathrm{~h}$ for further analysis or stored in distilled water.

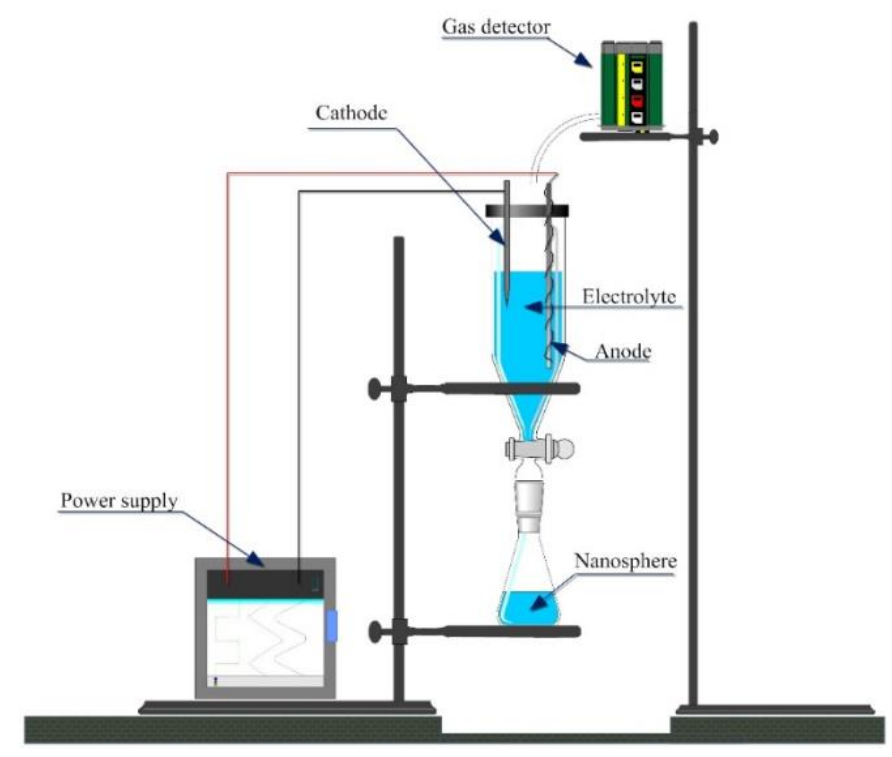

Fig. 1. Schematic diagram of submerged glow-discharge plasma.

\subsection{Characterization of iron wire and nanosphere}

The collected nanospheres from plasma process were analyzed via scanning electron microscope (SEM) technique to study the overall appearance of the produced nanostructures and to estimate the nanospheres sizes. SEM was performed using a JOEL JSM6010 PLUS/LV microscope operating at an accelerating voltage of $20 \mathrm{kV}$. The microscope also fitted with an Oxford Instrument INCA energy dispersive X-ray spectroscopy (EDS) attachment. EDS technique was used to detect the chemical elements of nanospheres and identify the metallic and oxidized phases at the nanospheres.

\section{Results and Discussion}

\subsection{Current - voltage (I-V) curves of submerged glow-discharge plasma}

Fig. 2 shows the typical I-V curves of different electrolyte concentration and Fig. 3 shows the I-V curves of vary cathode submerged length. Experiments carried out to measure the I$\mathrm{V}$ curves in Fig. 1 of scrap metal nail as cathode $(\varnothing 3 \mathrm{~mm})$ with constant submerged length $(0.5 \mathrm{~cm})$ in various electrolyte concentration $(1-0.01 \mathrm{M})$ of potassium carbonate. However, I$\mathrm{V}$ curves in Fig. 3 show the results from the experiment with various cathode submerged length $(0.5-1.5 \mathrm{~cm})$ in constant $0.01 \mathrm{M}$ of electrolyte. Temperature of electrolyte is crucial 
to initial the generating plasma and make it more effective at lower power density [21]. CO gas was detected during plasma initiation. The reactions occurred are described in 4 stages; (1) electrolysis stage, (2) vaporization stage, (3) transition stage, and (4) full plasma stage.

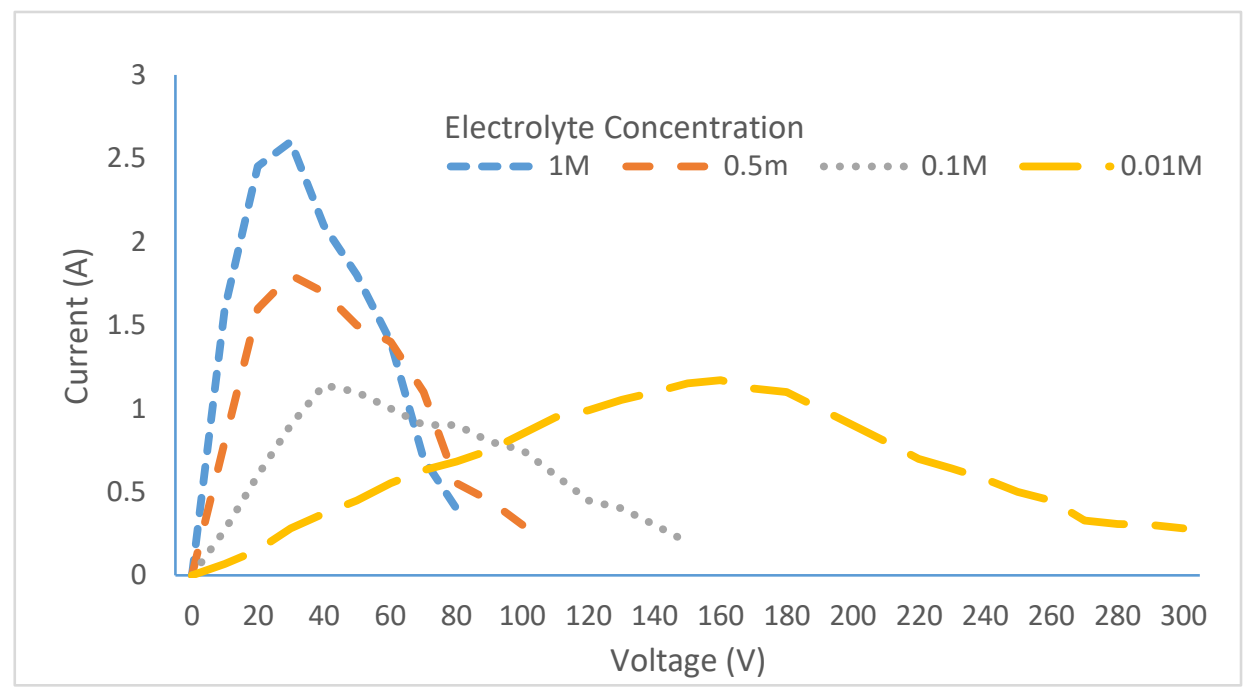

Fig. 2. Typical I-V curve for different electrolyte concentration.

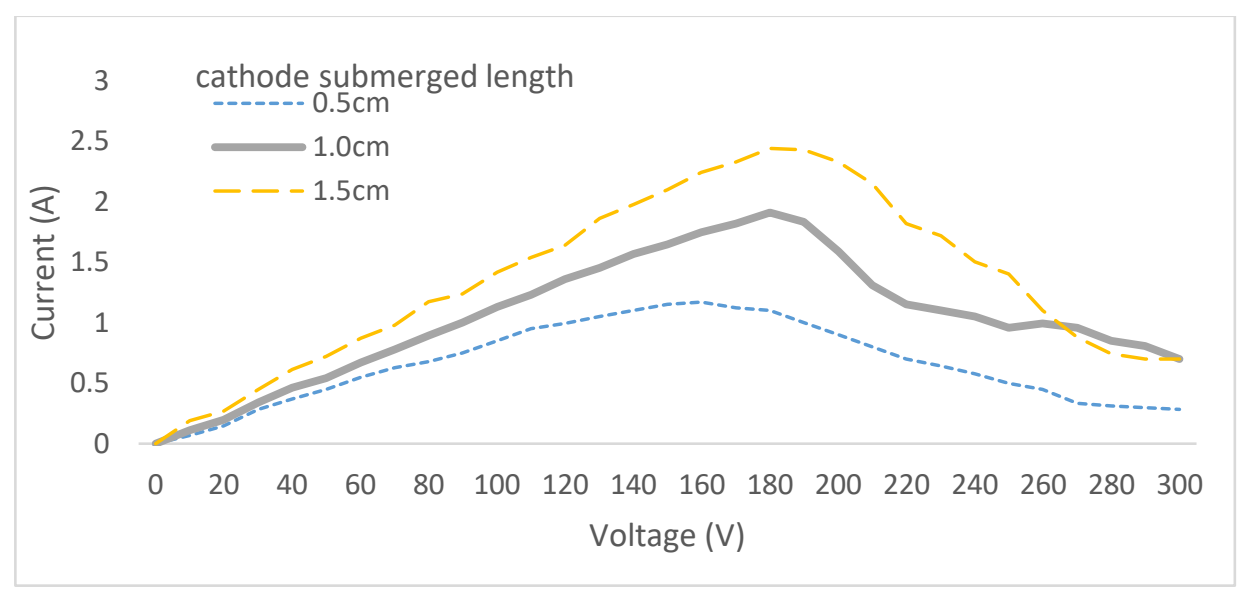

Fig. 3. Typical I-V curve for different cathode submerged length.

At low voltage of electrolysis stage (1), small bubbling was observed on both electrodes. During this stage, current increased along with voltage increase in accordance with Ohm's law. However, thermal loss was concentrated at the cathode side to heat up the electrolyte to boiling point during vaporization stage (2) with large bubbling sound. Hence, transition stage (3) with a thin layer of gas was surrounding the cathode and caused the conductivity to reduce, then the current reading start to reduce while fluctuating slightly. The collisions between electron and neutral atom produce excitation, ionization, dissociation with the emission of light. This phenomenon is known as "glow" discharge. The ions produced are accelerated toward the cathode, bombarding at the cathode surface, causing the release of secondary electrons. These secondary electrons causes more ionization collisions to ensure the glow-discharge as a self-sustained plasma [9]. Consequently, the discharge plasma can be generated at low current and make SGDP 
produce nanospheres with more economically.

During full plasma stage (4), the surface of the cathode is partially melted to produce nanoparticles owing to the concentration of current causes by the electro-thermal instability. [14]. Eventually, the cathode melts or ionizes at the spot to form nanoparticles by current concentration spot during discharge plasma, and rapidly cooled in the solution to form nanospheres due to surface tension and quenching effect of the electrolyte [16]. However, the amount of current measured was very much related to cathode submerged surface area and concentration of used electrolyte. Larger submerged area of cathode required more current to generate and maintain the plasma. Subsequently, this had an effect of increasing the nanosphere productivity.

\subsection{Micrograph of produced nanosphere}

Fig. 4 (a) is SEM of scrap metal at 850 times magnification and Fig. 5 (b), (c), (d) and (e) are iron oxide at 7000 times magnification produced at $0.01 \mathrm{M}, 0.1 \mathrm{M}, 0.5 \mathrm{M}$ and $1 \mathrm{M}$ of potassium carbonate concentration respectively after glow-discharge plasma synthesis and centrifuged to remove excessive electrolyte. SEM has been performed in order to confirm that the produced nanospheres have uniformly spherical shape due to the effect of surface tension and quenching of the molten metal by the surrounding electrolyte [22]. The particles were spherical in shape, with sizes ranging from $100 \mathrm{~nm}$ to $2000 \mathrm{~nm}$. Higher electrolyte concentrations were able to reduce the voltage applied to generate plasma in liquid.

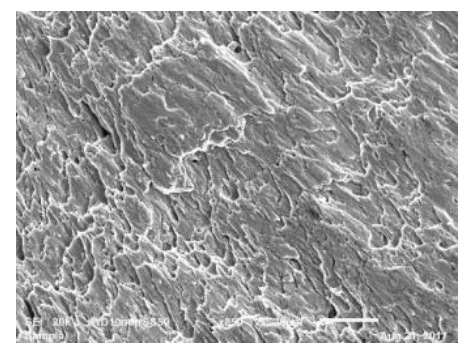

(a)

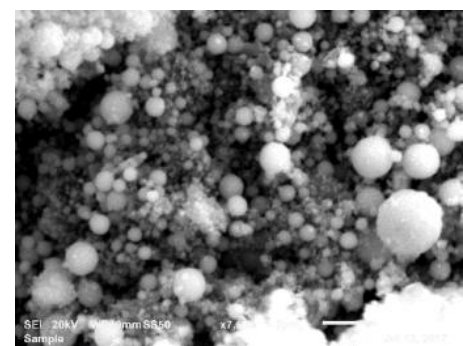

(b)

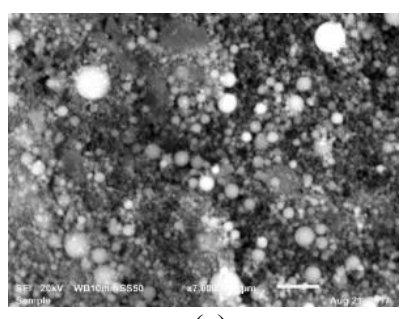

(c)

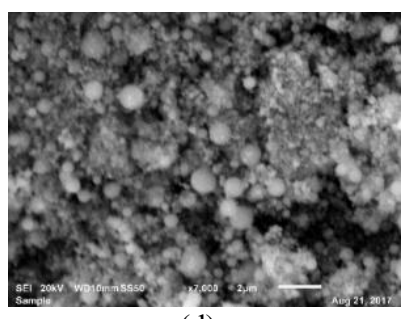

(d)

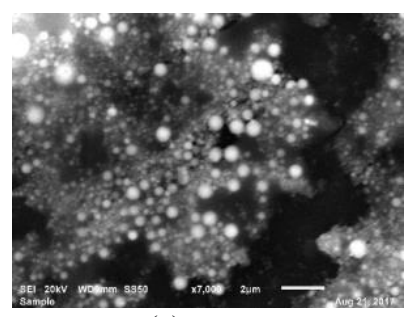

(e)

Fig. 6. SEM micrograph of (a) scrap metal, nanospheres produced from (b) $0.01 \mathrm{M}$, (c) $0.1 \mathrm{M}$, (d) $0.5 \mathrm{M}$ and (e) $1 \mathrm{M}$ of the electrolyte concentration.

\subsection{Elemental composition of produced nanosphere}

Table 1 shows the elemental composition of scrap metal nail and nanospheres produced via SGDP. Before the application of plasma, iron nail commonly consisted of Carbon (C) and iron $(\mathrm{Fe})$ atoms. Scrap metal initially showed around $6.45 \%$ and $93.55 \%$ of Carbon and 
Iron composition respectively. However, SGDP process has increased the Carbon content to $34-35 \%$ and Iron content has reduced to around $15-40 \%$. The nanospheres were found to contain Oxygen $(\mathrm{O})$ atom in addition to $\mathrm{C}$ and $\mathrm{Fe}$ atoms of its parent material. These results indicate that the surfaces of iron nanospheres were in their oxidised form after synthesis, and metal oxide produced from SGDP is commonly reported [16, 20]. Carbon content of the nanospheres produced from plasma process is much higher than raw scrap metal. Oxygen content is tended to increase with less concentration of electrolyte. EDS results also shown the higher percentage of Iron amount has remained with less concentration of potassium carbonate as electrolyte. It was found that at higher electrolyte concentration the plasma excitation voltage was reduced and do not have significant effect to the current flow. Current is proportionally related to submersion area of cathode.

Table 1. Elemental composition of the iron wire and nanosphere from plasma.

\begin{tabular}{lccccccc}
\hline \multirow{2}{*}{$\begin{array}{l}\text { Electrolyte } \\
\text { Concentration }\end{array}$} & $\begin{array}{l}\text { Voltage } \\
\text { plasma } \\
\text { maintained }\end{array}$ & $\mathrm{C}$ & $\mathrm{Fe}$ & $\mathrm{O}$ & Other & Total & \multirow{2}{*}{ Morphology } \\
\cline { 3 - 7 } & Scrap metal & 6.45 & 93.55 & - & $\mathrm{K}$ & 100 & \\
\hline \multirow{2}{*}{$1.0 \mathrm{M}$} & $80 \mathrm{~V}$ & 34.73 & 15.87 & 41.30 & 8.10 & 100 & Sphere \\
& & & & & $\mathrm{K}$ & 100 & Sphere \\
$0.5 \mathrm{M}$ & $100 \mathrm{~V}$ & 34.48 & 19.58 & 39.52 & 6.42 & & \\
$0.1 \mathrm{M}$ & $150 \mathrm{~V}$ & 34.52 & 23.92 & 40.26 & $\mathrm{~K}$ & 100 & Sphere \\
$0.01 \mathrm{M}$ & $280 \mathrm{~V}$ & 35.26 & 40.54 & 24.21 & - & 100 & Sphere \\
\hline
\end{tabular}

\subsection{The economics of nanospheres production using SGDP}

The price of a commercially available iron (II, III) oxide powder with $95 \%$ purity by Sigma-Aldrich is RM201.50/25g [23]. In order to perform a comparison, the calculation for the price of iron oxide nanospheres made using SGDP is shown in Equation 1.

SGDP Cost per 10g = Electricity cost per unit [24] + Electrolyte cost + Raw material cleaning cost

$$
\begin{aligned}
& =\mathrm{RM}(100 \mathrm{~V} * 1 \mathrm{~A} * 1 \text { hour*0.32) }+\mathrm{RM} 2+\mathrm{RM} 0.5 \\
& =\mathrm{RM} 34.5
\end{aligned}
$$

The equation 1 calculation corresponds to RM86.25 per $25 \mathrm{~g}$ of nanospheres. It is clear that the iron oxide nanospheres made using SGDP is 57\% cheaper compared to the cost of commercially available iron oxide powder. Therefore it shows that SGDP is an economical approach to nanomaterials production.

\section{Conclusions}

In this study, the effect of cathode submerged area and electrolyte concentration on plasma formation was studied. Nanospheres of Iron Oxide ranging from 100 to $2000 \mathrm{~nm}$ in size has been successfully produced. Higher electrolyte concentration was significantly reduce the initial plasma excitation voltage and further low electric power density ( $80 \mathrm{~V}$ and $0.3 \mathrm{~A})$ is required to generate nanospheres. As a conclusion, submerged glow-discharge plasma has the potential as a low-cost nanosphere synthesis method. 
The authors gratefully acknowledge the financial support provided by University College of Technology Sarawak under Grant scheme (Project No. UCTS/RESEARCH/<1/2016/03>(01)).

\section{References}

1. R.A. Khan, M.S. Liew, Z.B. Ghazali, Procedia - Procd Soc Behv. 109, 507-513, (2014).

2. S.K. Lachimpadi, J.J. Pereira, M.R. Taha, M. Mokhtar, Resour., Conserv. Recycl. 68, 96-103, (2012).

3. Z. Chen, J. Xu, Y. Chen, E.M. Lui, Constr. Build. Mater. 126, 641-660, (2016).

4. S.H. Hassan, N. Ahzahar, M.A. Fauzi, J. Eman, Procd Soc Behv. 42, 175-181, (2012).

5. R.A. Begum, C. Siwar, J.J. Pereira, A.H. Jaafar, Resour., Conserv. Recycl. 48, 86-98, (2006).

6. B. Peter, L. Christophe, Phys D Appl Phys. 42, 053001, (2009).

7. M.A. Malik, Plasma Chem. Plasma Process. 30, 21-31, (2010).

8. B. Jiang, J. Zheng, S. Qiu, M. Wu, Q. Zhang, Z. Yan, et al., Chem. Eng. J. 236, 348368, (2014).

9. T. Selma Mededovic, R.S. Gunnar, D. Fei, L.B. Christopher, M.H. Thomas, G.B. Douglas, et al., J Phys D Appl Phys. 50, 014003, (2017).

10. L. Wang, X. Jiang, Y. Liu, J Hazard Mater. 154, 1106-1114, (2008).

11. P.J. Hansen, M.P. Hoggard, A.C. Rathwell, J. Reprod. Immunol. 10, 157-166, (1987).

12. H.J. Forman, A. Bernardo, K.J.A. Davies, Arch. Biochem. Biophys. 603, 48-53, (2016).

13. N. Pourali, G. Foroutan, Phys. Lett. A. 380, 2422-2429, (2016).

14. M.R.M.B. Julaihi, S. Yatsu, M. Jeem, S. Watanabe, J. Exp. Nanosci. 10, 965-982, (2015).

15. T.A. Kareem, A.A. Kaliani, Ionics. 18, 315-327, (2012).

16. S. Yatsu, H. Takahashi, H. Sasaki, N. Sakaguchi, K. Ohkubo, T. Muramoto, et al. Arch. Metall. Mater. 58, 425, (2013)

17. G. Saito, Y. Nakasugi, T. Yamashita, T. Akiyama, Nanotechnology. 25, 135603, (2014).

18. G. Saito, Y. Nakasugi, T. Akiyama, J. Appl. Phys. 116, 083301, (2014).

19. G. Saito, Y. Nakasugi, T. Yamashita, T. Akiyama, Appl. Surf. Sci. 290, 419-424, (2014).

20. G. Saito, S. Hosokai, T. Akiyama, Mater. Chem. Phys. 130, 79-83, (2011).

21. G. Saito, Y. Nakasugi, T. Akiyama, J. Appl. Phys. 118, 023303, (2015).

22. Y. Nakasugi, G. Saito, T. Yamashita, T. Akiyama, J J. Appl. Phys.. 115, 123303, (2014).

23. https://www.sigmaaldrich.com/catalog/product/aldrich/310069?lang=en\&region=MY

24. https://www.sarawakenergy.com.my/index.php/customers/commercial-pricing-tariff 